\title{
Annual Meetings of JES
}

\begin{tabular}{|c|c|c|c|c|}
\hline & Dates & Cities & Presidents & Institutes \\
\hline $\begin{array}{l}1^{\text {st }} \\
2^{\text {nd }} \\
3^{\text {rd }} \\
4^{\text {th }} \\
5^{\text {th }} \\
6^{\text {th }} \\
7^{\text {th }} \\
8^{\text {th }} \\
9^{\text {th }} \\
10^{\text {th }} \\
11^{\text {th }} \\
12^{\text {th }} \\
13^{\text {th }}\end{array}$ & $\begin{array}{l}\text { Apr. 3, } 1927 \\
\text { Apr. 4-5, } 1928 \\
\text { Apr. 5-6, } 1929 \\
\text { Mar. 31, } 1930 \\
\text { Mar. 30, } 1931 \\
\text { Mar. 30, } 1932 \\
\text { Mar. 30, } 1933 \\
\text { Apr. 4, } 1934 \\
\text { Mar. 31, } 1935 \\
\text { Mar. 30, } 1936 \\
\text { Mar. 30, } 1937 \\
\text { Apr. 4, } 1938 \\
\text { Apr. 1, } 1939\end{array}$ & Kyoto & Prof. Kanji Tsuji & Kyoto University \\
\hline $\begin{array}{l}14^{\text {th }} \\
15^{\text {th }}\end{array}$ & $\begin{array}{l}\text { Mar. 28, } 1940 \\
\text { Mar. 29, } 1941\end{array}$ & Kyoto & Prof. Naohiko lizuka & Kyoto University \\
\hline $16^{\text {th }}$ & Mar. 29, 1942 & Tokyo & Prof. Ryuta Usui & The University of Tokyo \\
\hline $17^{\text {th }}$ & Mar. 29, 1943 & Kyoto & Prof. Kanji Tsuji & Kyoto University \\
\hline $18^{\text {th }}$ & Mar. 28, 1944 & Tokyo & Prof. Kozo Sakaguchi & National First Hospital \\
\hline $19^{\text {th }}$ & Apr., 1945 & Kyoto & Prof. Nobuyoshi Fuse & Osaka University \\
\hline $20^{\text {th }}$ & Apr. 1-2, 1947 & Osaka & Prof. Tomosaburo Ogata & The University of Tokyo \\
\hline $21^{\text {st }}$ & Apr. 1-2, 1948 & Tokyo & Prof. Kosaku Kakinuma & The University of Tokyo \\
\hline $22^{\text {nd }}$ & Apr. 1-2, 1949 & Kyoto & Prof. Shigeki Mori & Kyoto University \\
\hline $23^{\text {rd }}$ & Mar. 31-Apr. 1, 1950 & Tokyo & Prof. Akira Ogata & The University of Tokyo \\
\hline $24^{\text {th }}$ & Apr. 2-4, 1951 & Tokyo & Prof. Takayoshi Misawa & The University of Tokyo \\
\hline $25^{\text {th }}$ & Apr. 1-3, 1952 & Osaka & Prof. Nobuyoshi Fuse & Osaka University \\
\hline $26^{\text {th }}$ & Apr. 1-3, 1953 & Tokyo & Prof. Yosoji Ito & The University of Tokyo \\
\hline $27^{\text {th }}$ & Apr. 1-3, 1954 & Nagoya & Prof. Iwao Ogawa & Nagoya University \\
\hline $28^{\text {th }}$ & Apr. 2-5, 1955 & Kyoto & Prof. Shiku Takeishi & Kyoto Prefectural University of Medicine \\
\hline $29^{\text {th }}$ & Apr. 1-3, 1956 & Fukuoka & Prof. Goroo Kusunoki & Japanese Red Cross Fukuoka Hospital \\
\hline $30^{\text {th }}$ & Mar. 31-Apr. 2, 1957 & Tokyo & Prof. Shigeo Okinaka & The University of Tokyo \\
\hline $31^{\text {st }}$ & May 17-18, 1958 & Kyoto & Prof. Tadashi Miyake & Kyoto University \\
\hline $32^{\text {nd }}$ & Apr. 6-7, 1959 & Tokyo & Prof. Ken Nakao & The Jikei University \\
\hline $33^{\text {rd }}$ & Apr. 4-6, 1960 & Osaka & Prof. Tsuneo Yoshida & Osaka University \\
\hline $34^{\text {th }}$ & Apr.15-17, 1961 & Kanazawa & Prof. Fumio Akasu & Kanazawa University \\
\hline $35^{\text {th }}$ & Apr. 5-7, 1962 & Kobe & Prof. Shozo Tsuji & Kobe University \\
\hline $36^{\text {th }}$ & Apr. 3-4, 1963 & Osaka & Prof. Yoshio Adachi & Osaka University \\
\hline $37^{\text {th }}$ & Jun. 6-8, 1964 & Tokyo & Prof. Tokuji Ichikawa & The University of Tokyo \\
\hline $38^{\text {th }}$ & Apr. 9-11, 1965 & Fukuoka & Prof. Shibanosuke Katsuki & Kyushu University \\
\hline $39^{\text {th }}$ & Apr.11-13, 1966 & Tokyo & Prof. Takashi Kobayashi & The University of Tokyo \\
\hline $40^{\text {th }}$ & Apr. 4-5, 1967 & Nagoya & Prof. Kozo Yamada & Nagoya University \\
\hline $41^{\text {st }}$ & Apr. 5-7, 1968 & Kyoto & Prof. Hideo Yoshida & Kyoto Prefectural University of Medicine \\
\hline $42^{\text {nd }}$ & May 15-17, 1969 & Maebashi & Prof. Kojiro Shichijo & Gunma University \\
\hline $43^{\text {rd }}$ & Mar. 10-12,1970 & Osaka & Prof. Mitsuo Nishikawa & Osaka University \\
\hline
\end{tabular}




\begin{tabular}{|c|c|c|c|c|}
\hline & Dates & Cities & Presidents & Institutes \\
\hline $44^{\text {th }}$ & Apr. 3-4, 1971 & Tokyo & Prof. Kyoichiro Ochiai & Tokyo Medical and Dental University \\
\hline $45^{\text {th }}$ & Apr. 9-11, 1972 & Tokyo & Prof. Yawara Yoshitoshi & Hamamatsu University \\
\hline $46^{\text {th }}$ & Apr. 9-11, 1973 & Kyoto & Prof. Kozo Okamoto & Kyoto University \\
\hline $47^{\text {th }}$ & Apr. 8-10, 1974 & Tokyo & Prof. Fujio Yoshimura & The Jikei University \\
\hline $48^{\text {th }}$ & Jun. 2-4, 1975 & Kyoto & Prof. Masashi Fukase & Kyoto University \\
\hline $49^{\text {th }}$ & Jun. 6-7, 1976 & Tokyo & Prof. Kazuo Shizume & Tokyo Women's Medical University \\
\hline $50^{\text {th }}$ & May 17-19, 1977 & Fukuoka & Prof. Hiroshi Ibayashi & Kyushu University \\
\hline $51^{\text {st }}$ & Jun. 3-5, 1978 & Tokyo & Prof. Seiichi Matsumoto & Jichi Medical University \\
\hline $52^{\text {nd }}$ & Jun. 7-9, 1979 & Kyoto & Prof. Hiroo Imura & Kyoto University \\
\hline $53^{\text {rd }}$ & May 22-24, 1980 & Yokohama & Prof. Masazumi Kawakami & Yokohama City University \\
\hline $54^{\text {th }}$ & Jun. 4-6, 1981 & Okayama & Prof. Makoto Ofuji & Okayama University \\
\hline $55^{\text {th }}$ & May 28-30, 1982 & Tokyo & Prof. Minoru Irie & Toho University \\
\hline $56^{\text {th }}$ & May 26-28, 1983 & Osaka & Prof. Yuichi Kumahara & Osaka University \\
\hline $57^{\text {th }}$ & May 18-20, 1984 & Tokyo & Prof. Akira Kumagaya & Toyama Medical and Pharmaceutical University \\
\hline $58^{\text {th }}$ & May 14-16, 1985 & Nagoya & Prof. Naoki Kageyama & Nagoya University \\
\hline $59^{\text {th }}$ & May 14-16, 1986 & Sendai & Prof. Nobuaki Sasano & Tohoku University \\
\hline $60^{\text {th }}$ & Jun. 3-5, 1987 & Kyoto & Prof. Kanji Toritsuka & Fukui Medical University \\
\hline $61^{\text {st }}$ & Jul. 16, 1988 & Kyoto & Prof. Takuo Fujita & Kobe University \\
\hline $62^{\text {nd }}$ & Jun. 1-3, 1989 & Tokyo & Prof. Sho Yoshida & Chiba University \\
\hline $63^{\text {rd }}$ & May 17-19, 1990 & Osaka & Prof. Seiichiro Tarui & Osaka University \\
\hline $64^{\text {th }}$ & Jun. 1-3, 1991 & Tokyo & Prof. Etsuro Ogata & The University of Tokyo \\
\hline $65^{\text {th }}$ & May 28-30, 1992 & Tokushima & Prof. Shiro Saito & The University of Tokushima \\
\hline $66^{\text {th }}$ & Jun. 3-5, 1993 & Kanazawa & Prof. Ryoyu Takeda & Kanazawa University \\
\hline $67^{\text {th }}$ & Jun. 1-3, 1994 & Nagasaki & Prof. Shigenobu Nagataki & Nagasaki University \\
\hline $68^{\text {th }}$ & Jun. 1-3, 1995 & Tokyo & Prof. Hiroshi Demura & Tokyo Women's Medical University \\
\hline $69^{\text {th }}$ & Jul. 3-5, 1996 & Osaka & Prof. Mitsuo Inada & Kansai Medical University \\
\hline $70^{\text {th }}$ & Jun. 1-3, 1997 & Tokyo & Prof. Yoshimasa Shishiba & Toranomon Hospital \\
\hline $71^{\text {st }}$ & Jun. 4-6, 1998 & Fukuoka & Prof. Hajime Nawata & Kyushu University \\
\hline $72^{\text {nd }}$ & May 31-Jun. 2, 1999 & Yokohama & Prof. Toshikazu Saito & Jichi Medical University \\
\hline $73^{\text {rd }}$ & Jun. 16-18, 2000 & Kyoto & Prof. Yasuhiko Ibata & Kyoto Prefectural University of Medicine \\
\hline $74^{\text {th }}$ & Jun. 29-Jul. 1, 2001 & Yokohama & Prof. Takao Saruta & Keio University \\
\hline $75^{\text {th }}$ & Jun. 28-30, 2002 & Osaka & Prof. Yuji Matsuzawa & Osaka University \\
\hline $76^{\text {th }}$ & May 9-11, 2003 & Yokohama & Prof. Hisahiko Sekihara & Yokohama City University \\
\hline $77^{\text {th }}$ & Jun. 24-26, 2004 & Kyoto & Prof. Kazuwa Nakao & Kyoto University \\
\hline $78^{\text {th }}$ & Jul. 1-3, 2005 & Tokyo & Prof. Hiroshi Takami & Teikyo University \\
\hline $79^{\text {th }}$ & May 19-21, 2006 & Kobe & Prof. Kazuo Chihara & Kobe University \\
\hline $80^{\text {th }}$ & Jul. 14-16, 2007 & Tokyo & Prof. Toshiro Fujita & The University of Tokyo \\
\hline $81^{\text {st }}$ & May 16-18, 2008 & Aomori & Prof. Toshihiro Suda & Hirosaki University \\
\hline $82^{\text {nd }}$ & Apr. 23-25, 2009 & Maebashi & Prof. Masatomo Mori & Gunma University \\
\hline $83^{\text {rd }}$ & Mar. 25-28, 2010 & Kyoto & Prof. Akira Teramoto & Nippon Medical School \\
\hline
\end{tabular}

